\title{
Moon Landing Trajectory Optimization
}

\author{
Ibrahim Mustafa MEHEDI \\ Center of Excellence in \\ Intelligent Engineering Systems (CEIES) \\ King Abdulaziz University, Jeddah 21589, Saudi Arabia \\ AND \\ Electrical and Computer Engineering Department \\ King Abdulaziz University, Jeddah 21589, Saudi Arabia \\ Email: imehedi@kau.edu.sa
}

\author{
Md. Shofiqul ISLAM \\ Electrical and Computer Engineering Department \\ King Abdulaziz University \\ Jeddah 21589, \\ Saudi Arabia
}

\begin{abstract}
Trajectory optimization is a crucial process during the planning phase of a spacecraft landing mission. Once a trajectory is determined, guidance algorithms are created to guide the vehicle along the given trajectory. Because fuel mass is a major driver of the total vehicle mass, and thus mission cost, the objective of most guidance algorithms is to minimize the required fuel consumption. Most of the existing algorithms are termed as "near-optimal" regarding fuel expenditure. The question arises as to how close to optimal are these guidance algorithms. To answer this question, numerical trajectory optimization techniques are often required. With the emergence of improved processing power and the application of new methods, more direct approaches may be employed to achieve high accuracy without the associated difficulties in computation or pre-existing knowledge of the solution. An example of such an approach is DIDO optimization. This technique is applied in the current research to find these minimum fuel optimal trajectories.
\end{abstract}

Keywords-lunar landing; trajectory optimization; optimization techniques; DIDO optimization

\section{INTRODUCTION}

Return to the moon becomes a demanding issue. A lot of scientists and engineers confirmed considerable interests in the past couple of decades [1, 2, 3, 7, 8, 9]. Two-dimensional solution for lunar descent and landing from orbital speed conditions is presented in [4]. The same guidance scheme is improved to 3-dimensional point of view in [6]. More precise 3- Dimensional advanced solution of lunar descent and landing is discussed in [5]. Trajectory optimization capabilities during the Apollo era were severely limited by computing power. An analytic solution for the one-dimensional vertical terminal descent of a lunar soft-landing, based on an application of Pontryagin's minimum principle, was found by Meditch [10] in 1964. Meditch showed the existence of an optimum thrust program that achieves soft landing under powered descent. Extensive numerical research on the one-dimensional problem was performed by Teng and Kumar [11], using various cost functionals. Their method is based on a time transformation, applied to the calculus of variations. The solution was found numerically, using a quasi-linearization method. In 1971, Shi and Eckstein [12] derived an exact analytic solution for the problem which Teng and Kumar addressed. With the increase in computing power, trajectory optimization techniques, of the type to be discussed in Section 3, have greatly increased the feasibility of generating optimal trajectories with higher complexity and applicability. Recently, Vasile and
Floberghagen [13] applied a Spectral Elements in Time (SET) approach to the lunar soft-landing problem. Within the work, a lunar landing descent from three parking orbit scenarios down to an altitude of $2[\mathrm{~m}]$ above the surface was optimized. The cost function used was based on the square of the control input, which has been noted to be different from the minimum fuel solution [14]. The optimization method used to explore the Moon landing problem in the current research is the Legendre Pseudospectral Method. The Legendre Pseudospectral Method has been applied to a variety of trajectory optimization problems, including problems of ascent guidance [15], satellite formation flying [16], and impulsive orbit transfers [17]. DIDO [18] is used to implement the Legendre Pseudospectral Method in the current research which is discussed in this paper.

The rest of present paper is arranged as follow: The optimization theory has been presented in Section II. The methods of optimization have been explained in Section III. DIDO optimization is introduced in Section IV. The Section V Illustrates the vehicle specification for Moon landing. The Section VI and VII demonstrate the trajectory results and optimized responses with discussion. The conclusion has been given in Section VIII.

\section{OPTIMIZATION THEORY}

To maximize or minimize a specified criterion is the task of an optimization problem. This criterion is a function of the parameter which is referred to as a cost function. On this cost function, optimization takes place. Let $x \in \Re^{n}$ be a vector of parameters and let $J(x): \Re^{n} \rightarrow \Re$ define a cost function. It is desired to find the value $\mathrm{x}^{*}$ which minimizes $\mathrm{J}$ out of all admissible $\mathrm{x}$. The global minimum is defined as:

$$
J\left(x^{*}\right) \leq J(x) \text { for all admissible } \mathrm{x}
$$

A local minimum is defined as:

$$
J\left(x^{*}\right) \leq J(x) \text { for all } \mathrm{x} \text { in the neighborhood of } \mathrm{x}^{*}
$$

In a globally convex system, any local optimum found is also the global optimum. On the other hand if the function is non-convex, finding a local optimum does not necessarily imply that it is also the global optimum solution. Fig. 1 illustrates the concept of convexity vs. non-convexity and local vs. global optimums for an arbitrary one-dimensional function $f(x)$. 

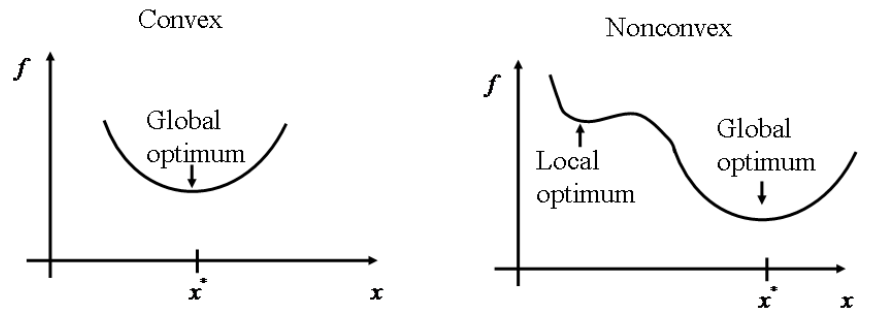

Fig. 1. One-dimensional convexity

Only one optimal solution which is the global optimum is available in the example shown for convexity. Two optimal, local and global optimum solutions exist in the non-convex example. Most trajectory optimization problems are nonconvex, and therefore, only local optimums are readily found.

A parameter optimization problem that has a linear cost function, linear constraints, and has only real values is known as a linear programming problem. The term is only used for problems which have real values; otherwise, it falls into the category of integer or mixed-integer programming. If the problem has only real values but includes a nonlinear cost function or nonlinear constraints, it is referred to as a nonlinear programming (NLP) problem.

From basic calculus, recall that a local minimum of a function, which is the function of one variable (i.e., $f(x)$ ), can be found by locating a point where the first derivative of the function on the variable equals zero and the second derivative is positive. This notion is expanded to higher dimensions with gradients and Hessians. A gradient is a vector consisting of the first-order partial derivatives of a function on each variable. The Hessian is a matrix consisting of the second-order partial derivatives of the function. For example, the following conditions are satisfied at a local minimum for the cost function $J(x)$ :

$$
\begin{aligned}
& \frac{\partial J}{\partial x}=0 \\
& \frac{\partial^{2} J}{\partial x^{2}} \geq 0
\end{aligned}
$$

One could attempt to determine the minimum of the cost function analytically, but it may be difficult or impossible. Therefore, iterative techniques are used to locate the minimum by searching over the region of admissible $x$. Newton's method, the most common technique, uses the gradient and Hessian information at the current location to determine a search direction. There are numerous other iterative techniques used, most being based on the principle of Newton's method.

\section{A. Constrained Optimization}

Constrained optimization problem can be either equality constraints or inequality constraints. While a function, or functions, of the parameters, equal to a specific value, then it is added to the optimization problem as an equality constraints. The parameters can vary, but relationships between the parameters remain fixed. An equality constraint has the form:

$$
\text { where } f: \Re^{n} \rightarrow \Re^{q} f(x)=0
$$

Formulating the augmented cost function, the most convenient method of solving equality constrained optimization problem, which is a combination of the cost function and the constraints with multipliers.

$$
J^{\prime}=J(x)+\lambda^{T} f(x)
$$

To find the minimum, the gradient of $J^{\prime}$ is taken on $x$ and $\lambda$ and set to zero. The first-order necessary conditions for optimality become:

$$
\begin{aligned}
& \frac{\partial J^{\prime}}{\partial x}=\frac{\partial J}{\partial x}+\frac{\partial f}{\partial x} \lambda=0 \\
& \frac{\partial J^{\prime}}{\partial \lambda}=f(x)=0
\end{aligned}
$$

On the other hand, an inequality constraint has the form:

$$
\text { where } \mathrm{g}: \mathfrak{R}^{n} \rightarrow \mathfrak{R}^{q} g(x) \leq 0
$$

The constraints are adjoined to the cost function in a similar manner as the equality constraints, but with the multipliers $\boldsymbol{\mu}$. The augmented cost function is now defined as follows:

$$
J^{\prime}=J(x)+\lambda^{T} f(x)+\mu^{T} g(x)
$$

Inequality constraints can be categorized into either 'active' or 'inactive' classes. The vector $\mathbf{g}(\mathrm{x})$ can be written as seen in (11), with $\mathrm{g}_{\mathrm{i}}(\mathrm{x})$ for $(\mathrm{i}=1 \ldots \mathrm{p})$ representing individual components of the p-dimensional vector. An active constraint is when a component of the constraint vector equals zero at the optimum solution, (i.e., $\mathrm{g}_{\mathrm{i}}\left(\mathrm{x}^{*}\right)=0$ ). The optimization problem is bounded by these constraints. Inactive constraints are constraints where $\mathrm{g}_{\mathrm{i}}\left(\mathrm{x}^{*}\right)<0$ and these constraints do not affect the optimal solution. The equation $\boldsymbol{\mu}^{\mathrm{T}} \mathrm{g}=0$, which is known as complementary slackness, ensures that the individual inequality constraints are either active or do not affect the solution.

$$
g=\left[\begin{array}{c}
g_{1}(x) \\
\cdot \\
\cdot \\
\cdot \\
g_{p}(x)
\end{array}\right]
$$

Karush-Kuhn-Tucker (KKT) conditions are named for the first-order necessary optimality conditions. These are given by (8) and (9), and the following:

$$
\begin{aligned}
& \frac{\partial J \prime}{\partial x}=0 \\
& \mu \geq 0 \\
& \mu^{T} g=0
\end{aligned}
$$

\section{B. Concept of optimal control}

A particular category of optimization problems which comprise dynamical constraints that vary with time is recognized as functional optimization problems, where the term "functional" is used to denote a function of a function. Functional optimization problems that have an input, or control, to be determined are identified as an optimal control problems. Optimal control problems have an extensive diversity of applications, together with the field of trajectory optimization.

Let $x \in \Re^{n}$ be the state of a continuous system where $t \in \mathfrak{R}$ is time. Furthermore, let $u(t) \in \mathfrak{R}^{n}$ be the control of input. Lastly, let the dynamical constraints that govern the change of $x(t)$ with respect to time be given as: 


$$
\dot{x}=f(x(t), u(t), t)
$$

To search for the function $\mathbf{u}(t)$ that minimize the cost functional, $J$ is a general optimal control problem, with the condition that the constraints imposed on the problem. This problem should include the dynamical constraints. It is normally desired to either minimize a functional of the state and control over the entire time span or the final value of a criterion. The cost functional is therefore composed of two parts: a terminal cost $\emptyset: \mathfrak{R}^{n} \times \mathfrak{R} \rightarrow \mathfrak{R}^{n}$ (commonly referred to as the Mayer cost) and an integrated cost $L: \mathfrak{R}^{n} \times \mathfrak{R}^{m} \times$ $\mathfrak{R} \rightarrow \mathfrak{R}$ (known as the Lagrange cost). The Bolza form of the cost functional, as seen in (16), is a combination of the terminal and integrated costs.

$$
J=\varnothing\left(x\left(t_{f}\right), t_{f}\right)+\int_{t_{0}}^{t_{f}} L(x(t), u(t)) d t
$$

The initial and final boundary conditions that the system must satisfy are given by (17) and (18), respectively.

$$
\begin{aligned}
& \varphi_{0}\left(x\left(t_{0}\right), t_{0}\right)=0 \\
& \left.\varphi_{f} x\left(t_{f}\right), t_{f}\right)=0
\end{aligned}
$$

Where $\varphi_{0}: \Re^{n} \times \mathfrak{R} \rightarrow \mathfrak{R}^{q 0}$ and $\varphi_{f}: \mathfrak{R}^{n} \times \mathfrak{R} \rightarrow \mathfrak{R}^{q f}$.

Augmented cost functional $J^{\prime}$ is formulated to combine all of this information. A vector of Lagrange multipliers is called the costate. This costate adjoined by the dynamical and terminal constraints to the cost functional. Here, $\lambda(t) \epsilon \mathfrak{R}^{n}$ is Lagrange multipliers, and $v \in \mathfrak{R}^{q f}$ is terminal constraint multipliers. The equation given below is similar in Reference [19].

$$
\begin{aligned}
J^{\prime} & =\varnothing\left(x\left(t_{f}\right), t_{f}\right)+v^{T} \varphi\left(x\left(t_{f}\right), t_{f}\right) \\
& +\int_{t_{0}}^{t_{f}}\left[L(x(t), u(t), t)+\lambda(t)^{T}(f(x(t), u(t), t)-\dot{x})\right] d t
\end{aligned}
$$

Hamiltonian and end point functional are defined in (20) and (21), respectively to simplify the augmented cost functional. $J^{\prime}$ is simplified to (22). The functional dependencies have been omitted for clarity.

$$
\begin{aligned}
& H=L+\lambda^{T} f \\
& G=\varnothing+v^{T} \varphi \\
& \quad J^{\prime}=G+\int_{t_{0}}^{t_{f}}\left[H-\lambda^{T} \dot{x}\right] d t
\end{aligned}
$$

It is determined a stationary point of the augmented cost functional using calculus of variations. Using the previous concept, the variation of $J^{\prime}$ is taken and appropriate functions are set to zero. The resulting necessary conditions for a local minimum for the free final time problem are derived by Hull [20] as:

$$
\begin{aligned}
& \dot{x}=\frac{\partial H}{\partial \lambda}=f(x, u, t) \\
& \dot{\lambda}=-\frac{\partial H}{\partial x} \\
& 0=\frac{\partial H}{\partial u} \\
& \varphi_{0}\left(x\left(t_{0}\right), t_{0}\right)=0 \\
& \left.\varphi_{f} x\left(t_{f}\right), t_{f}\right)=0 \\
& \lambda^{T}\left(t_{f}\right)=\left(\frac{\partial G}{\partial x}\right)_{t=t_{f}} \\
& (H)_{t=t_{f}}=-\left(\frac{\partial G}{\partial x}\right)_{t=t_{f}}
\end{aligned}
$$

\section{OPTIMIZATION METHODS}

Optimality conditions described in the previous are to be solved to find a stationary point. An analytical solution is almost impossible for most non-linear optimization problems. Therefore, computer-aided numerical methods are the alternate way in finding the optimal solution. There are numerous numerical methods that have been formulated to solve optimal control problems, and it is not in the scope of this thesis to explore them all. The method used in this research is the Legendre Pseudospectral Method, which is a direct transcription method that uses a spectral technique. The meanings of 'direct', 'transcription', and 'spectral' will be discussed below.

\section{A. Direct Methods}

Computer aided numerical methods can be divided into two distinct categories: direct and indirect. An indirect method uses information from the costate differential, the maximum principle, and the boundary conditions shown in (26 - 29), to find the optimal solution. To use this method, an estimate of the costate is required a priori, which may pose a problem since the costate does not usually have physical significance.

The direct method is commonly used to optimize directly the cost function such as (16). The method searches for the feasible region for a minimum of the cost function starting from an initial guess of the state and control. It is reported as a local minimum solution because it is impossible to search the entire feasible region. If it can be proven that the problem is convex, then local optimality implies global optimality. Being familiar with the dynamics of the problem, it can provide an initial guess of the state and control rather than costate. In some cases, a simple propagation of the state from the desired initial conditions with no control input is sufficient. However, if the problem is highly non-convex, a good initial guess may be crucial in finding the correct local optimal solution.

\section{B. Direct Transcription Methods}

The direct method implies some different ways such as direct shooting methods, direct transcription, etc. A direct transcription method is used in this research and described as follows.

Transformation of continuous time domain system into discrete time domain system is an important issue in real-time optimal control problem to be implemented on a computer. The locations in time at which the problem is discretized are referred to as 'nodes', and can be uniformly or non-uniformly distributed in the time domain. At each node, the discrete system represents the continuous system, and links must be made between the nodes to represent the dynamics of the original continuous system. A transcription method is used to transform the continuous system into the discrete problem. Most optimal control problems include either a nonlinear cost function or nonlinear constraints (which may include nonlinear dynamical constraints) that are only functions of real variables. As a result, the transcribed problem is an NLP.

Two consecutive tasks are performed by this transcription method. One is to transform the problem from a continuous system to a discrete system, and another is to link the nodes together in a way that represents the dynamics of the original 
problem. Representing the dynamics of a continuous system is done by different ways such as the Euler method, the RungeKutta method, and spectral methods. The method used in this research is a spectral method, which fits globally orthogonal polynomials to the discrete data over the entire time span. The Legendre Pseudospectral Method uses a special class of orthogonal polynomials, known as Legendre Polynomials. The interior nodes are placed at the roots of the Legendre polynomial derivative, known as the Legendre-Gauss-Lobatto (LGL) points, which provides higher accuracy in the results.

\section{DIDO OPTIMIZATION}

In the past, it was an extremely difficult task to analyze fully a complex system involving numerous dependent variables. It was an indirect and approximate method to allow solutions to be found. But now computers have improved exponentially in processing speed and ability, allowing complex computations to be completed quickly and efficiently without imposing a significant drain on resources. One example of this improvement is in the field of optimization analysis. With the emergence of such improved processing power and the application of new methods, more direct approaches may be employed to achieve high accuracy without the associated difficulties in computation or pre-existing knowledge of the solution. An example of such an approach is DIDO optimization. The method is named for Queen Dido of Carthage (circa 850BC) who was the first person known to have solved a dynamic optimization problem.

DIDO relies on the Legendre Pseudospectral Method, which has been developed and employed primarily in fluid flow modeling. This method employs Legendre Polynomials to create an approximation of variables over multiple nodes as opposed to the use of a fixed order polynomial. This allows, despite discontinuities in the governing equations, a solution to be attained with high accuracy which also satisfies the imposed optimization criterion, where most direct methods do not [21]. The result is a method of solving a complex dynamic optimization problem without a priori knowledge of the solution or incredibly complex analytic computations. This is exactly the type of method required to optimize a problem as complex as an examination of the trade space for lunar landing.

The DIDO optimization code requires the development of five different files to specify the problem accurately. Once the problem is defined, DIDO optimizes a solution using the restrictions found in the problem definition and on the cost function as previously discussed. These files include the Cost Function File, Dynamics-Function File, Events File, Path File and Problem File [22].

\section{VEHICLE SPECIFICATION}

The vehicle is assumed to be axially symmetric with a thrust to mass ratio that varies between 4.0 [N/kg] and 10.0 $[\mathrm{N} / \mathrm{kg}]$. It assumed that the vehicle has one throttle-able main engine, which is fixed to the vehicle and does not gimbal. For a hypothetical initial mass of 1800 [kg] (which is representative of an unmanned vehicle), a value of 8000 [N] was chosen for the engine's maximum thrust limit, $\mathrm{F}_{\max }$. The engine's exhaust velocity, $\mathrm{V}_{\mathrm{ex}}$, was chosen as $3500[\mathrm{~m} / \mathrm{s}]$, which corresponds to a specific impulse of $358 \mathrm{~s}$.
In comparison, the Surveyor vehicles had final landed masses varying from $1431[\mathrm{~kg}]$ to 1486 [kg] [23, 24]. Each lander had a propulsion system which consisted of one main solid propellant retro-fire engine and three throttlable liquid propellant vernier engines. The vernier engines, which were used to remove the final $100[\mathrm{~m} / \mathrm{s}]$ of velocity and also for attitude control during the retro-fire, were capable of providing $133[\mathrm{~N}]$ to $463[\mathrm{~N}]$ of thrust each. They had a specific impulse which varied from 273 [s] at minimum thrust to 287 [s] at maximum thrust [25].

\section{TRAJECTORY RESULTS}

This section explores the results obtained during trajectory optimization of the Moon landing problem. The equations of motion derived in References [4, 5, 6,] were coded in DIDO. In this section, all work is limited to planar motion, but can easily be extended to three-dimensions. The convexity of this problem was not determined; therefore, all results are reported as local optimal solutions.

An initial parking orbit $100[\mathrm{~km}]$ was chosen for the analysis throughout this simulation. The objective of the current research is to minimize fuel usage while meeting specified operational constraints. The cost function was chosen to be the final mass of the vehicle because this is the most direct measurement of fuel usage, as seen in (30).

$$
J_{\min }=-m\left(t_{f}\right)
$$

The main criterion is to land with almost zero velocity at the surface while using bounded thrust.

\section{A. Boundary Conditions}

The vehicle parameters, as described in Section 5, are listed along with the initial and end conditions in Table 1 and 2.

TABLE I. InITIAL CONDITIONS AND VeHICle PARAMETERS

\begin{tabular}{|l|l|l|}
\hline Parameters & Value & Unit \\
\hline Maximum available thrust & 8000 & {$[\mathrm{~N}]$} \\
\hline Specific Impulse & 358 & {$[\mathrm{~s}]$} \\
\hline Initial altitude & 100 & {$[\mathrm{~km}]$} \\
\hline Initial down range distance & 0 & {$[\mathrm{~km}]$} \\
\hline Initial velocity vector pitch angle & 90 & {$[\mathrm{deg}]$} \\
\hline
\end{tabular}

TABLE II. END CONDITIONS OF POWERED DESCENT CONDITIONS

\begin{tabular}{|l|l|l|}
\hline Parameters & Value & Unit \\
\hline Final altitude & 1 & {$[\mathrm{~km}]$} \\
\hline Final down range distance & 425 & {$[\mathrm{~km}]$} \\
\hline Final velocity vector pitch angle & 0.1 & {$[\mathrm{deg}]$} \\
\hline Lunar gravitation acceleration & 1.623 & {$\left[\mathrm{~m} / \mathrm{s}^{2}\right]$} \\
\hline
\end{tabular}

\section{RESPONSES}

Initially, it is assumed that the expected landing point is about $425[\mathrm{~km}]$ far. Other initial and end conditions are dictated in Table 1 and 2. The physical path of the trajectory is shown in Fig. 2. The path solved for by DIDO and depicted in the Fig. is the most efficient in terms of fuel consumption, and thus is the optimal solution regarding this analysis. 


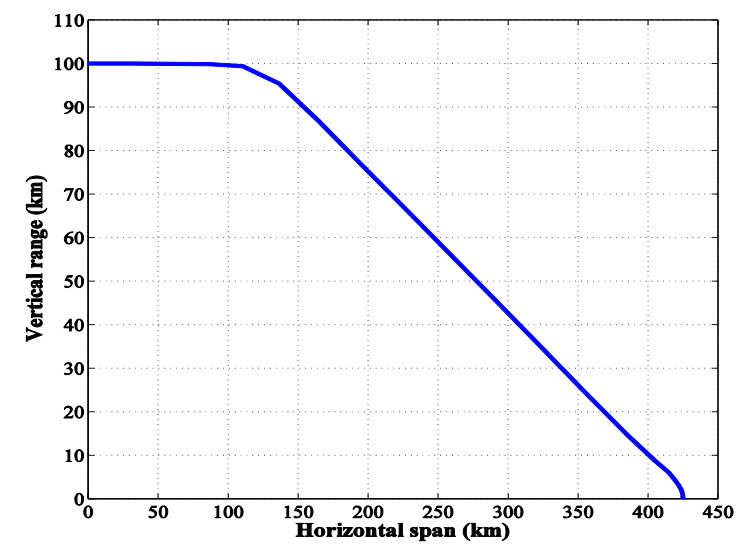

Fig. 2. Optimized lunar landing trajectory

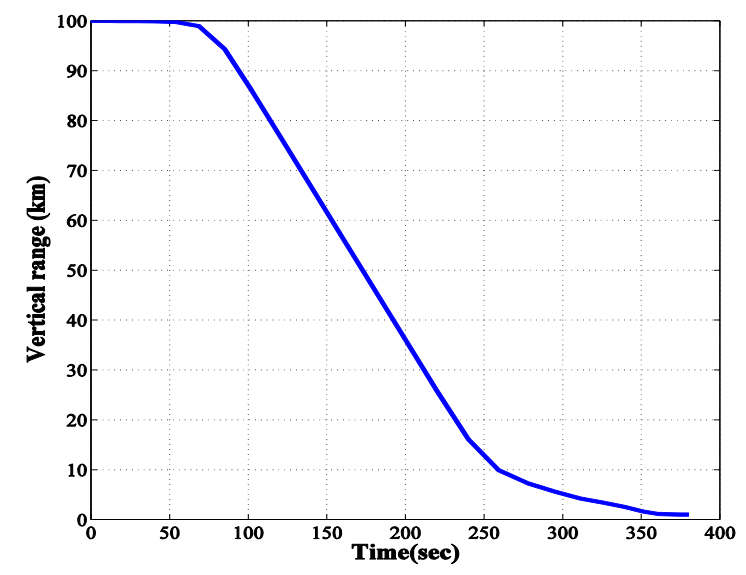

Fig. 3. Optimized lunar landing trajectory: Vertical range

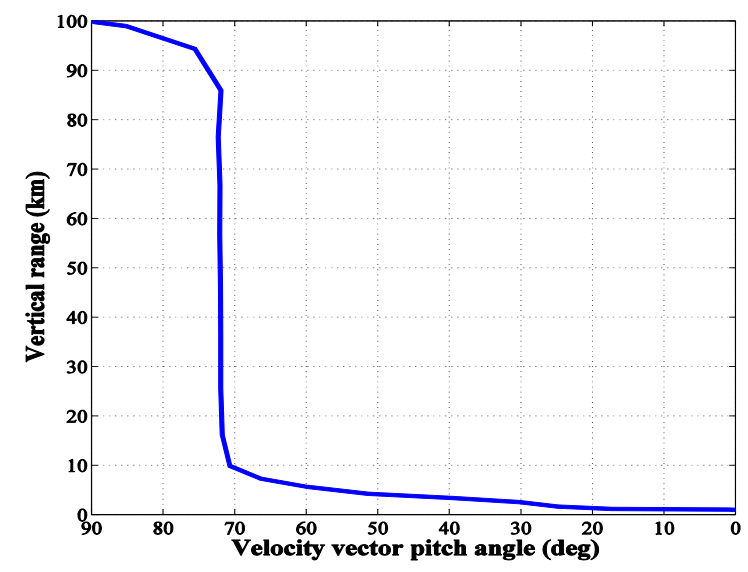

Fig. 4. Optimized lunar landing trajectory: Vertical range as a function of velocity

To instill a better understanding of the trajectory, the vertical and horizontal positions relative to time are displayed in Fig.s 3 and 5. Further, trajectory profiles regarding vertical and horizontal positions as a function of velocity vector pitch angle are shown in Fig.s 4 and 6. Fig. 3 illustrates the parametric shape of the burn that takes place, indicating features of perfect fuel optimization. The burn is timed precisely, in this case, to minimize the amount of fuel consumed.

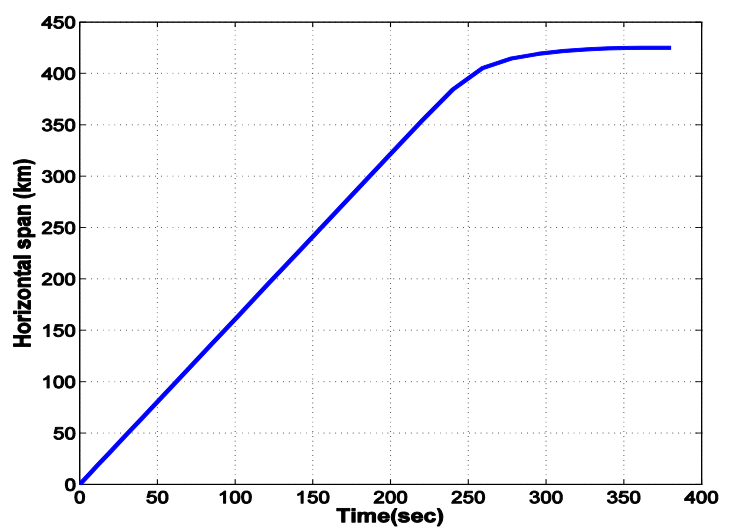

Fig. 5. Optimized lunar landing trajectory: Horizontal span

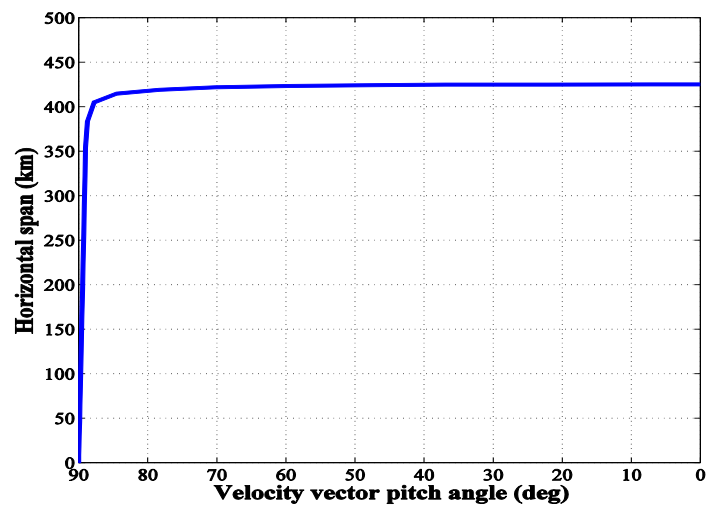

Fig. 6. Optimized lunar landing trajectory: Horizontal span as a function velocity vector pitch angle

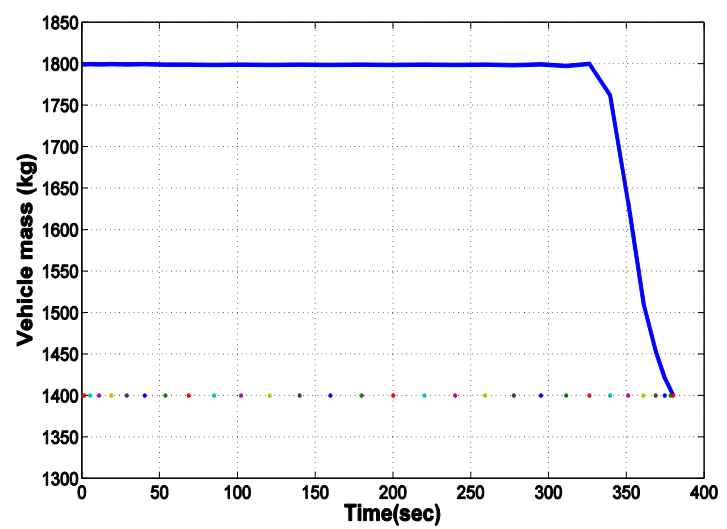

Fig. 7. Optimized lunar landing trajectory: Vehicle mass

Fig. 5 demonstrates the nearly constant progression in the horizontal plane. Another parameter of interest is that which drives the optimization, mass, and is shown in Fig. 7. It is observed that the $400[\mathrm{~kg}]$ fuel was used to minimize the amount of addition fuel being carried. The intent is that this approximately simulates the eventual fuel budget of the lunar 
landing vehicle though safety reserves and other factors will affect the final value. When compared to Fig. 3 it can be seen that the main usage of fuel occurs during the vertical braking burns, as would be expected due to a large amount of thrust required during these periods. Movement in the horizontal plane causes this line to continuously decrease, but at a much slower rate, due to the relatively small amount of thrust in this direction.

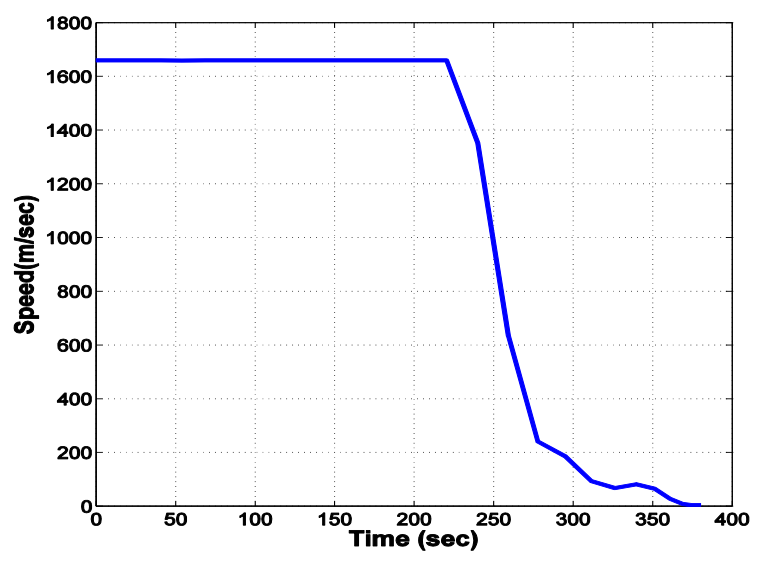

Fig. 8. Optimized lunar landing trajectory: Vehicle speed

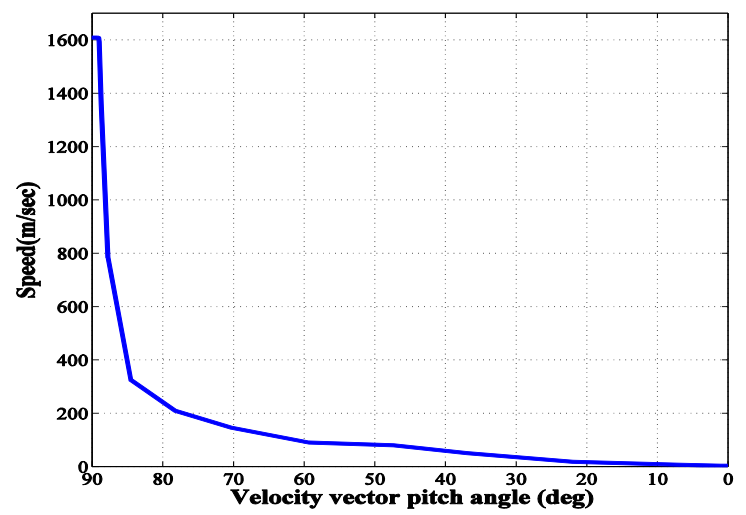

Fig. 9. Optimized lunar landing trajectory: Vehicle speed as a function of velocity vector pitch angle

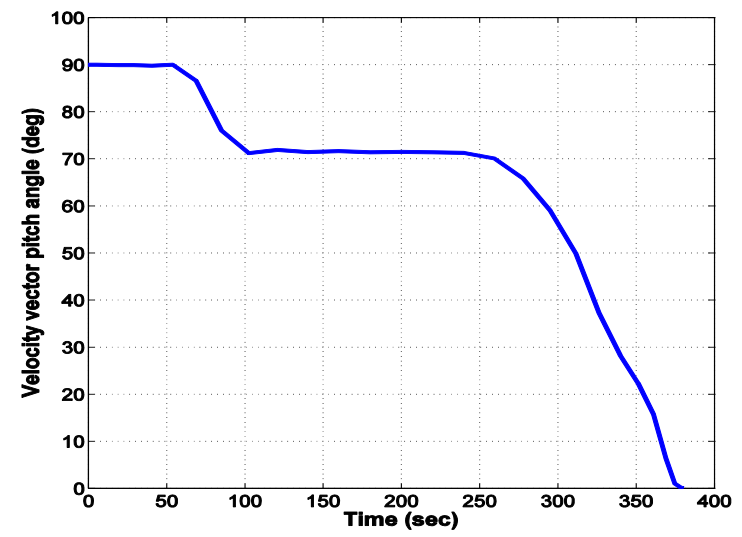

Fig. 10. Optimized lunar landing trajectory: Velocity vector pitch angle

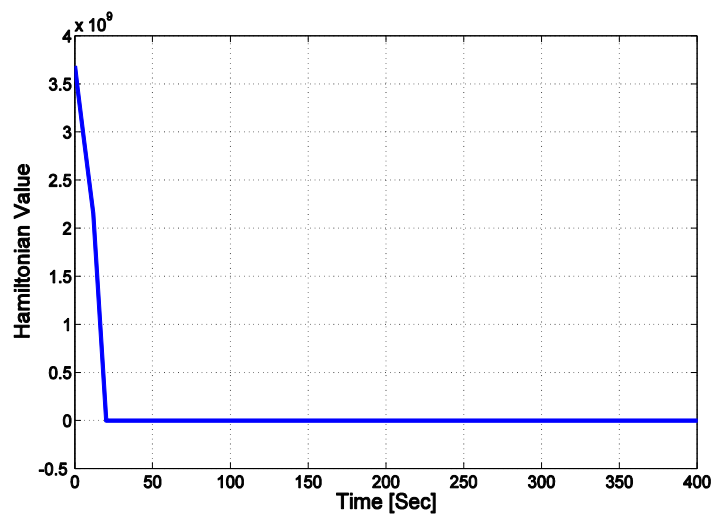

Fig. 11. Hamiltonian evaluation for optimality verification

Details of trajectory, including vehicle speed against time and velocity vector pitch angle are shown in Fig. 8 and 9, respectively. It is important to note the difference in y-axis scales on the vehicle speed plots. This trajectory is largely horizontal, having a maximum velocity of 1609 [m/s] which decreases rapidly once the final braking burn commences. Again, the velocity vector pitch angle is plotted against time in Fig. 10.

\section{CONCLUSION}

According to the Minimum Principle, given a candidate optimal solution, there exists a collection of dual functions and variables such that certain statements are true. Depending upon the problem these statements are examined as a means to test the optimality of the DIDO run. From the Minimum Principle, it must satisfy the Hamiltonian Minimization Condition [26, 27], Therefore, the current investigation with the help of DIDO includes the results of Hamiltonian. This is plotted in Fig. 11. The Hamiltonian should be constant over the entire time span. Looking at the Fig. 11, the estimate of the Hamiltonian is fairly small, but oscillation is present. A large fluctuation is seen where the throttle switch occurs, because of the initialization in the control during descent start. Thus, the computer simulation has indeed found the exact optimal solution at a very high precision. Using this direct approach like DIDO optimization helped to achieve high accuracy without the associated difficulties in computation or pre-existing knowledge of the solution.

\section{ACKNOWLEDGMENT}

This article was funded by the Deanship of Scientific Research (DSR), King Abdulaziz University, Jeddah. Therefore, the authors acknowledge with thanks DSR financial support

\section{REFERENCES}

[1] R. Cheng, "Lunar terminal guidance In Lunar Missions and Exploration," edited by C. T. Leondes and R. W. Vance, Univ. of California Engineering and Physical Sciences Extension Series, Wiley, New York, pp. 308-355, 1964.

[2] R. Cheng, C. Meredith and D. Conrad, "Design considerations for survevor guidance," Journal of Spacecraft and Rockets, Vol. 3, Issue 11, p. 1569-1576, 1966.

[3] R. Klumpp, "Apollo guidance, navigation, and control: Apollo lunar-descent guidance," Technical report, MIT Charles Stark Draper Laboratory, 1971. 
[4] I. M. Mehedi and T. Kubota, "Advanced guidance scheme for lunar descent and landing from orbital speed conditions," Transaction of Japan Society for Aeronautical and Space Sciences, Vol. 54, Issue 184, pp. 98105, 2011.

[5] I. M. Mehedi, T. Kubota and U. M. Al-Saggaf UM, "3- Dimensional advanced solution of lunar descent and landing," Journal of Vibroengineering, Vol. 17, Issue 1, pp.527-543, 2015.

[6] I. M. Mehedi, T. Kubota and U. M. Al-Saggaf UM, “3- Dimensional Analytical solution for lunar descent scheme," Iranian Journal of Science and Technology Transactions of Electrical Engineering, Vol. 39, Issue E1, pp. 67-77, 2015.

[7] R. Sostaric, "Lunar descent reference trajectory," Technical report, NASA/JSC, 2006.

[8] S. Ueno and Y. Yamaguchi, "Near-minimum guidance law of a lunar landing module," 14th IFAC Symposium on Automatic Control in Aerospace, pp. 377-382, 1998.

[9] L. Xing-Long, D. Gaung-Ren and T. Kok-Lay, "Optimal soft landing control for moon lander," Automatica, Vol. 44, pp. 1097-1103, 2008.

[10] J. S. Meditch, "On the Problem of Optimal Thrust Programming for a Lunar Soft Landing," IEEE Transactions on Automatic Control, Vol. AC-9, No. 4, pp. 484, 1964.

[11] L. Teng and K. S. P. Kumar, "Optimum Control for Lunar Soft Landing," Astronautica, Acta, Vol. 13, No. 5-6, pp. 545, 1968.

[12] Y. Y. Shi and M. C. Eckstein "An Exact Solution For Optimum Controlled Soft Lunar Landing," Vol. 16, No. 1, pp. 18, 1971.

[13] M. Vasile and R. Floberghagen, "Optimal Trajectories for Lunar Landing Missions," AAS/GSFC International Symposium, Advances in the Astronautical Sciences, Greenbelt, MD, pp. 281-295, 1998.

[14] I. M. Ross, "How to Find Minimum-Fuel Controllers," AIAA Guidance, Navigation, and Control Conference and Exhibit, American Institute of Aeronautics and Astronautics, Inc., Providence, RI, pp. 1-10, AIAA Paper 2004-5346, 2004.

[15] Rea, J. R. A Legendre Pseudospectral Method for Rapid Optimization of Launch Vehicle Trajectories, Master's thesis, Massachusetts Institute of Technology, Cambridge, MA, May 2001.
[16] S. I. Infeld, S. B. Josselyn and W. Murray, "Design and Control of Libration Point Spacecraft Formations," AIAA Guidance, Navigation, and Control Conference and Exhibit, Providence, RI, pp. 1-14, AIAA Paper 2004-4786, 2004.

[17] S. A. Stanton, "Optimal Orbital Transfer using a Legendre Pseudospectral Method," Master's thesis, Massachusetts Institute of Technology, 2003.

[18] I. M. Ross, “User's Manual for DIDO (Ver. PR.13): A MATLAB Application Package for Solving Optimal Control Problems," Technical Report 04-01.0, Naval Postgraduate School, Monterey, CA, February 2004.

[19] A. Bryson and Y. C. Ho, "Applied Optimal Control-Optimization, Estimation and Control," Hemisphere Publishing Company, 1975.

[20] D. Hull, "Optimal Control Theory for Applications," Mechanical Engineering Series, Springer-Verlag New York, Inc., New York, NY, 2003.

[21] M. Ross, and F. A. Fahroo, "Perspective on Methods for Trajectory Optimization," AIAA/AAS Astrodynamics Specialist Conf., Monterey, 2002.

[22] H. B. Robert and A. C. Antoulas, "Nonlinear Approach to Aircraft Tracking Problem," Journal of Guidance, Control, and Dynamics, 17(5), pp. 1124-1130, October 1994.

[23] Surveyor Project Final Report, Part 2. Project "Description and Performance,” Tech. Rep. JPL-TR-32-1265, NASA, Pasadena, CA, July 1969.

[24] "Surveyor I Mission Report, Part 1. Mission Description and Performance," Tech. Rep. JPL-TR-32-1023, NASA, Pasadena, CA, August 1966.

[25] M. E. Ellion, H. DiCristina, A. R. Maffei, and A. Briglio, "Development of the Surveyor Vernier Propulsion System (VPS)," AIAA Second Propulsion Joint Specialist Conference, Colorado Springs, CO, 1966.

[26] R. Vinter, “Optimal Control,” BirkhAauser, Boston, MA 2000

[27] I. M. Ross, "Control and Optimization: An Introduction to its Principles and Applications," Electronic Edition, Naval Postgraduate School, Monterey, CA, October 2006. 